\title{
Pandemia de coronavírus (COVID-19): o que os fonoaudiólogos devem saber
}

Letter to the Editor

Andressa Silva Freitas ${ }^{1}$ (1)

Guilherme Maia Zica ${ }^{1}$ (1)

Christiane Lopes de Albuquerque ${ }^{2}$ (1)

\section{Coronavirus pandemic (COVID-19): what speech therapists should know}

O mundo vive perplexo o surto de uma nova síndrome respiratória aguda grave. O novo coronavírus (COVID-19), relatado em dezembro de 2019, teve seu epicentro em Wuhan, província de Hubei, na República Popular da China e se espalhou para diversos países em todos os continentes ${ }^{(1)}$.

Em 30 de janeiro de 2020, a Organização Mundial de Saúde (OMS) declarou o surto de COVID-19 como a sexta emergência de saúde pública de interesse internacional após a H1N1 (2009), poliomielite (2014), Ebola na África Ocidental (2014), Zika (2016) e Ebola no República Democrática do Congo (2019). Portanto, trabalhadores da saúde, governos e a população em geral precisam cooperar globalmente para impedir sua propagação ${ }^{(1,2)}$. Em 11 de fevereiro de 2020, o Grupo de Estudos sobre Coronavírus do Comitê Internacional de Taxonomia de Vírus o designou como coronavírus 2 da síndrome respiratória aguda grave (SARS-CoV-2) com base na filogenia, taxonomia e prática estabelecida. Logo após, a OMS nomeou a doença causada por este coronavírus como Doença de Coronavírus 2019 (COVID-19) ${ }^{(1)}$.

Com base nos dados atuais, há a hipótese de que o SARS-CoV-2 possa ter se hospedado inicialmente em morcegos e ter sido transmitido aos seres humanos via pangolim ou outros animais selvagens vendidos no mercado de frutos do mar de Huanan na China ${ }^{(2)}$.

O coronavírus é um ácido ribonucleico envolto e de fita simples, nomeado por sua aparência de coroa solar devido a picos de superfície de 9 a $12 \mathrm{~nm}$ de comprimento ${ }^{(3)}$. Estudos apontam que sua principal via de transmissão é por meio do contato e gotículas respiratórias ${ }^{(2)}$.

O sinal clínico inicial da doença, que permitiu a detecção e diferenciação dos casos, foi a pneumonia. As observações até o momento sugerem um período médio de incubação viral de cinco dias ${ }^{(4)}$. Em pacientes sintomáticos, as manifestações clínicas da doença geralmente começam em menos de uma semana (variando de zero a 20 dias), consistindo em febre, tosse, congestão nasal, fadiga e outros sinais de infecções do trato respiratório superior. Relatórios mais recentes também descrevem sintomas gastrointestinais e infecções assintomáticas, especialmente entre crianças pequenas ${ }^{(5)}$.

A proporção de indivíduos infectados por COVID-19 que permanecem assintomáticos durante o curso da infecção ainda não foi definitivamente estimada, porém há relatos de que $80 \%$ dos casos podem se apresentar sem sintomas ${ }^{(6)}$.

Evidências informais estão se acumulando rapidamente em sites de todo o mundo de que a anosmia e a disgeusia podem ser sintomas associados à infecção por COVID-19. A anosmia, em particular, tem sido observada em pacientes assintomáticos com resultados positivos para o coronavírus, e, portanto, pode ser utilizada como ferramenta de rastreio. No dia 22 de março, a Academia Americana de Otorrinolaringologia - Cirurgia de Cabeça e Pescoço (AAO-HNS) publicou em seu site informações sugerindo que a anosmia, a hiposmia e a disgeusia, na ausência de outras doenças respiratórias, como rinite alérgica,

\footnotetext{
Endereço para correspondência:

Andressa Silva de Freitas

Seção de Cirurgia de Cabeça e

Pescoço, Instituto Nacional do Câncer

- INCA

Praça da Cruz Vermelha, Rio de Janeiro (RJ), Brasil, CEP: 20230-130

E-mail: andressa.freitas@inca.gov.br
}

\footnotetext{
Trabalho realizado no Laboratório Interdisciplinar de Cabeça e Pescoço - LICEP, Instituto Nacional de Câncer - INCA - Rio de Janeiro (RJ), Brasil.

${ }^{1}$ Instituto Nacional do Câncer - INCA, Ministério da Saúde - Rio de Janeiro (RJ), Brasil.

${ }^{2}$ Universidade Estadual do Rio de Janeiro - UERJ - Rio de Janeiro (RJ), Brasil.

Fonte de financiamento: nada a declarar.

Conflito de interesses: nada a declarar.
} 
rinossinusite aguda ou rinossinusite crônica, deveriam alertar os médicos para a possibilidade de infecção por COVID-19 e justificar séria consideração pelo isolamento e teste desses indivíduos ${ }^{(7)}$.

A infecção pode progredir para um maior comprometimento com dispneia e sintomas torácicos correspondentes a pneumonia grave em aproximadamente $15 \%$ dos pacientes ${ }^{(8,9)}$. A pneumonia ocorre principalmente na segunda ou terceira semana de uma infecção sintomática. Sinais proeminentes de pneumonia viral incluem diminuição da saturação de oxigênio, desvios de gases no sangue, alterações visíveis por meio de raios $\mathrm{X}$ do tórax e outros exames de imagem ${ }^{(10)}$.

A taxa de detecção de casos muda diariamente e pode ser rastreada em tempo quase real por diversos meios de comunicação. Vários estudos começaram a ser escritos e publicados, a grande maioria com base em observações de dados do surto inicial na China, descrevendo a população que foi acometida pela doença. São estudos retrospectivos e a grande maioria apresenta casuísticas com vieses de seleção devido à falta de critérios científicos que a pandemia impõe. Os estudos compartilham relatos de idade mediana de 56 anos, alta porcentagem de homens (62\%) e quase metade de pacientes com comorbidades $(48 \%)^{(4)}$. Entre os pacientes adultos, a febre foi o sintoma relatado de forma mais comum $(92,8 \%)$, seguido de tosse $(69,8 \%)$, dispneia $(34,5 \%)$, mialgia $(27,7 \%)$, cefaleia $(7,2 \%)$ e diarreia $(6,1 \%)$. Observou-se rinorreia em apenas 4\%, dor de garganta em 5,1\% e dor na região da faringe em $17,4 \%$ dos pacientes com informações clínicas relevantes $^{(4)}$.

A síndrome da insuficiência respiratória aguda é uma complicação da infecção pelo vírus COVID-19, mais comum em idosos, com baixa imunidade e associação de comorbidades, destacando-se a hipertensão, diabetes, doença arterial coronariana, bronquite, alterações isquêmicas do sistema nervo central e doença de Parkinson. Os fatores de risco para complicações pulmonares e aumento da taxa de óbito são o aumento da idade e o acúmulo de comorbidades ${ }^{(11)}$.

Se a insuficiência respiratória não puder ser melhorada ou piorar continuamente em um curto período de tempo, é consenso entre os relatos de que a intubação deve ser realizada imediatamente. Estima-se que aproximadamente 5\% dos pacientes se tornam críticos e, portanto, necessitam de intubação orotraqueal e ventilação invasiva ${ }^{(5)}$.

Pode-se observar que a ventilação invasiva através de um tubo orotraqueal é comum em meio a esse surto e é atualmente considerada a melhor estratégia ventilatória com o isolamento mais efetivo da via aérea (menor propagação do vírus). Este procedimento foi realizado em 2,3\% dos 1.099 pacientes com COVID-19 confirmados, com base na coorte de pacientes de 552 hospitais em 30 províncias, regiões autônomas e municípios da China $^{(4)}$. Já há relatos na literatura sobre a recuperação pulmonar dias após o tratamento, onde se observa a diminuição da extensão das lesões, acompanhada de consolidação parcial e fibrose no órgão ${ }^{(12)}$.

Considerando que os idosos são a população com maior risco de complicações, que o tratamento de escolha instituído para os casos graves até o momento é a ventilação invasiva com chance de sequelas pulmonares, o contexto caracteriza-se como de risco para a disfagia. O grau da disfunção pode variar a depender de diversos fatores como a idade do paciente, a associação ou não com um quadro de presbifagia, tempo de intubação orotraqueal e outras comorbidades, que podem justificar alterações de deglutição prévias e a extensão da fibrose pulmonar no pós-tratamento.

Há aproximadamente 140 anos já é possível observar a preocupação com os comprometimentos provenientes de uma intubação orotraqueal inadequada e/ou prolongada ${ }^{(13)}$. A literatura descreve como prolongado um período superior a 48 horas, o que representa um maior risco para o desenvolvimento de transtornos da deglutição ${ }^{(14)}$. Na China, constatou-se que a ventilação invasiva nos pacientes portadores de COVID-19 foi mantida em média por aproximadamente 12 dias $^{(4)}$

A passagem do tubo por via oral pela orofaringe e laringe e o uso de bloqueadores neuromusculares ou agentes sedativos durante o período de ventilação mecânica podem originar diversas alterações: modificações na anatomia glótica, atrofia ou inatividade dos músculos esqueléticos responsáveis pela deglutição, alterações nos quimiorreceptores e mecanorreceptores presentes nas mucosas da faringe e laringe, déficits no reflexo de tosse e sensibilidade intraoral ${ }^{(13-16)}$.

Estas alterações patológicas oriundas da intubação orotraqueal podem ser agravadas em indivíduos $\operatorname{idosos}^{(3,16)}$. A perda de peso e massa muscular, alterações na coluna cervical, redução da mobilidade e elasticidade dos tecidos, redução da produção de saliva, comprometimento do estado dentário, sensibilidade oral e faríngea reduzidas, função olfativa e gustativa reduzidas são aspectos do envelhecimento que se somaam ao quadro de disfagia proveniente do tratamento da síndrome respiratória ${ }^{(15,17)}$.

A literatura afirma que a alteração da deglutição após a intubação orotraqueal é prevalente e requer avaliação e acompanhamento especializados, e que as taxas de disfagia e broncoaspiração nessa população é subestimada ${ }^{(14,16,17)}$. Sendo assim, é fundamental compreendermos que há uma grande parcela de pacientes pós-COVID-19 que necessitarão de atendimento fonoaudiológico, considerando a necessidade absoluta de via aérea artificial em doentes graves somados ou não a comorbidades.

Após a recuperação, os indivíduos portadores de COVID-19 apresentarão algum grau de comprometimento pulmonar ${ }^{(12)}$. Sabe-se que doenças respiratórias como fibrose pulmonar e apneia obstrutiva do sono estão intimamente associadas a déficits patológicos da coordenação da deglutição e respiração, aspecto de risco para disfagia e broncoaspiração ${ }^{(18,19)}$.

É evidente que doentes graves portadores de COVID-19 com necessidade de intubação orotraqueal não têm indicação de acompanhamento fonoaudiológico durante o período de ventilação mecânica, porém é factível de que a maioria necessitará de avaliação e acompanhamento especializado para o tratamento de seus déficits de deglutição após o período crítico. A presbifagia, intubação oral prolongada e possíveis comprometimentos pulmonares são fatores de risco para o desenvolvimento de disfagia neste grupo.

No planejamento terapêutico para a reabilitação destes pacientes, é necessário ter atenção a todas as medidas de precaução padrão, com uso dos equipamentos de proteção individual adequados 
para proteção da pele e mucosas, que estão sendo orientados amplamente por órgãos normativos.

Especificamente no aspecto terapêutico, os procedimentos devem ser cautelosamente escolhidos a fim de minimizar a formação de aerossóis. Já há relatos de que as células dos tecidos orais, especialmente as células epiteliais da língua, podem fornecer possíveis rotas de risco potencial da infecção pelo COVID-19(20). Ainda não há relatos seguros sobre o tempo médio em que ocorre a infecção aguda pelo COVID-19 e também sobre o tempo que é necessário para que o paciente esteja livre do vírus. Sendo assim, até que os exames não detectem mais a presença do vírus, é aconselhável que as estratégias de reabilitação excluam a manipulação direta da mucosa da cavidade oral, que sejam evitados procedimentos como desinsuflar o cuff, tosse, assim como uso de dispositivos que possam aumentar a possibilidade de contaminação (laringoscopia e endoscopia por exemplo).

Para os pacientes em reabilitação ambulatorial que necessitam permanecer em quarentena, a necessidade de proteger a equipe de saúde é uma das principais preocupações quando a manutenção da reabilitação é vital. Neste contexto, um programa de terapia universal, que possa ser estendido por maiores períodos e que possa ter continuidade em casa, evitando assim o atendimento contínuo, é aconselhável. Na falta desta possibilidade, há ainda a opção da telerreabilitação, que tem a vantagem adicional de manter a reabilitação, com supervisão remota, sem risco de exposição ao vírus. Neste contexto, o Conselho Federal de Fonoaudiologia publicou em seu site, no dia 17 de março, orientações sobre teleatendimento, considerando as condições emergenciais, que podem ser utilizadas durante os meses de março e abril de 2020 ou enquanto durar a pandemia ${ }^{(21)}$.

Neste momento, em que cada leito hospitalar é essencial e a alta de pacientes o mais precoce possível é vital para reduzir a taxa de contaminação, o trabalho da Fonoaudiologia é fundamental. $\mathrm{O}$ fonoaudiólogo deve avaliar e viabilizar a atuação em pacientes com prioridades clínicas, para possibilitar a alta hospitalar precoce e segura, investigando a biomecânica da deglutição e orientando a melhor estratégia quanto à reabilitação e à reintrodução da alimentação oral em consonância com a evolução do quadro, sabendo respeitar os limites impostos por uma patologia que ainda é pouco conhecida e favorecendo o pronto reestabelecimento do paciente, auxiliando a alta, tão importante neste momento em que a falta de vagas em leitos hospitalares pode ser a principal causa de mortalidade.

\section{REFERÊNCIAS}

1. Velavan TP, Meyer CG. The COVID-19 epidemic. Trop Med Int Health. 2020;25(3):278-80. http://dx.doi.org/10.1111/tmi.13383. PMid:32052514.

2. Jin YH, Cai L, Cheng ZS, Cheng H, Deng T, Fan YP, et al. A rapid advice guideline for the diagnosis and treatment of 2019 novel coronavirus (2019$\mathrm{nCoV}$ ) infected pneumonia (standard version). Mil Med Res. 2020;7(1):4. http://dx.doi.org/10.1186/s40779-020-0233-6. PMid:32029004.

3. Wu D, Wu T, Liu Q, Yang Z. The SARS-CoV-2 outbreak: what we know. Int J Infect Dis. 2020;94:44-8. http://dx.doi.org/10.1016/j.ijid.2020.03.004. PMid:32171952.

4. Guan WJ, Ni ZY, Hu Y, Liang WH, Ou CQ, He JX, et al. Clinical characteristics of coronavirus disease 2019 in China. N Engl J Med. 2020;382(18):1708-20. http://dx.doi.org/10.1056/NEJMoa2002032. PMid:32109013.
5. Lai CC, Shih TP, Ko WC, Tang HJ, Hsueh PR. Severe acute respiratory syndrome coronavirus 2 (SARS-CoV-2) and corona virus disease-2019 (COVID-19): the epidemic and the challenges. Int J Antimicrob Agents. 2020;105924(3):55. http://dx.doi.org/10.1016/j.ijantimicag.2020.105924. PMid:32081636.

6. Ling Z, Xu X, Gan Q, Zhang L, Luo L, Tang X, et al. Asymptomatic SARS$\mathrm{CoV}-2$ infected patients with persistent negative CT findings. Eur J Radiol. 2020;126:108956. http://dx.doi.org/10.1016/j.ejrad.2020.108956. PMid:32199142.

7. AAO-HNS: American Academy of Otolaryngology - Head and Neck Surgery. Anosmia, hyposmia, and dysgeusia symptoms of coronavirus disease. Alexandria: AAO-HNS COVID-19 Resources; 2020. [citado em 2020 Mar 24]. Disponível em: https://www.entnet.org/content/coronavirus-disease-2019-resources

8. Yang W, Cao Q, Qin L, Wang X, Cheng Z, Pan A, et al. Clinical characteristics and imaging manifestations of the 2019 novel coronavirus disease (COVID-19): a multi-center study in Wenzhou city, Zhejiang, China. J Infect. 2020;80(4):38893. http://dx.doi.org/10.1016/j.jinf.2020.02.016. PMid:32112884.

9. Yuen KS, Ye ZW, Fung SY, Chan CP, Jin DY. SARS-CoV-2 and COVID-19: the most important research questions. Cell Biosci. 2020;10(1):40. http://dx.doi. org/10.1186/s13578-020-00404-4. PMid:32190290.

10. Meng L, Qiu H, Wan L, Ai Y, Xue Z, Guo Q, etal. Intubation and Ventilation amid the COVID-19 Outbreak: wuhan's experience. Anesthesiology. 2020;132(6):131732. http://dx.doi.org/10.1097/ALN.0000000000003296. PMid:32195705.

11. Bouadma L, Lescure FX, Lucet JC, Yazdanpanah Y, Timsit JF. Severe SARS-CoV-2 infections: practical considerations and management strategy for intensivists. Intensive Care Med. 2020;46(4):579-82. http://dx.doi.org/10.1007/ s00134-020-05967-x. PMid:32103284.

12. Xu YH, Dong JH, An W, Lv XY, Yin XP, Zhang JZ, et al. Clinical and computed tomographic imaging features of Novel Coronavirus Pneumonia caused by SARS-CoV-2. J Infect. 2020;80(4):394-400. http://dx.doi.org/10.1016/j. jinf.2020.02.017. PMid:32109443.

13. Macewen W. Clinical observations on the introduction of tracheal tubes by the mouth, instead of performing tracheotomy or laryngotomy. BMJ. 1880;2(1022):163-5. http://dx.doi.org/10.1136/bmj.2.1022.163. PMid:20749636.

14. Brown CV,Hejl K, Mandaville AD, Chaney PE, Stevenson G, Smith C. Swallowing dysfunction after mechanical ventilation in trauma patients. JCrit Care. 2011;26(1):108 e9-13. http://dx.doi.org/10.1016/j.jcrc.2010.05.036. PMid:20869841.

15. Macht M, Wimbish T, Clark BJ, Benson AB, Burnham EL, Williams A, et al. Postextubations dysphagia is persistent and associated with poor outcomes in survivors of critical illness. Crit Care. 2011;15(5):231. http://dx.doi.org/10.1186/ cc10472. PMid:22017882.

16. El Gharib AZG, Berretin-Felix G, Rossoni DF, Seiji Yamada S. Effectiveness of therapy on post-extubation dysphagia: clinical and electromyographic findings. Clin Med Insights Ear Nose Throat. 2019;12. http://dx.doi. org/10.1177/1179550619873364. PMid:31548797.

17. Burns HP, Dayal VS, Scott A, Van Nostrand AWP, Bryce DP. Laryngotracheal trauma: observations on its pathogenesis and its prevention following prolonged orotracheal intubation in the adult. Laryngoscope. 1979;89(8):1316-25. http:// dx.doi.org/10.1002/lary.1979.89.8.1316. PMid:459664.

18. Ghannouchi I, Marie JP, Duclos C, Verin E. Alteration of swallowing and ventilation coordination in respiratory diseases in small mammals. Dysphagia. 2020;35(2):308-13. http://dx.doi.org/10.1007/s00455-019-10024-x. PMid:31227886.

19. Losurdo A, Brunetti V, Broccolini A, Caliandro P, Frisullo G, Morosetti R, et al Dysphagia and obstructive sleep apnea in acute, first-ever, ischemic stroke. J Stroke Cerebrovasc Dis. 2018;27(3):539-46. http://dx.doi.org/10.1016/j. jstrokecerebrovasdis.2017.09.051. PMid:29074066.

20. Xu H, Zhong L, Deng J, Peng J, Dan H, Zeng X, et al. High expression of ACE2 receptor of 2019-nCoV on the epithelial cells of oral mucosa. Int J Oral Sci. 2020;12(1):8. http://dx.doi.org/10.1038/s41368-020-0074-x. PMid:32094336.

21. Choon-Huat Koh G, Hoenig H. How should the rehabilitation community prepare for 2019-nCoV? Arch Phys Med Rehabil. 2020;101(6):1068-71. http://dx.doi.org/10.1016/j.apmr.2020.03.003. PMid:32194034.

\section{Contribuição dos autores}

ASF participou da idealização do estudo, interpretação dos dados e redação do artigo; GMZ participou da idealização do estudo, análise e interpretação dos dados e redação do artigo; CLA revisão do manuscrito. 